\title{
Comparison of the Effects of Magnesium Sulfate and Dexmedetomidine on Operation Field of Candidates for Endoscopic Sinus Surgery
}

\author{
Ebrahim Khoshraftar¹, Mohammad Hossein Bakhshaei ${ }^{2}$, Mehdi Shahabinejad ${ }^{3}$, Jalal Porolajal ${ }^{4}$, \\ Farnaz Hashemian ${ }^{5, *}$ \\ ${ }^{1}$ Assistant Professor, Department of Anesthesiology, School of Medicine, Hamadan University of Medical Sciences, Hamadan, Iran \\ ${ }^{2}$ Associate Professor, Department of Anesthesiology, School of Medicine, Hamadan University of Medical Sciences, \\ Hamadan, Iran \\ ${ }^{3}$ Resident, Department of Anesthesiology, School of Medicine, Hamadan University of Medical Sciences, Hamadan, Iran \\ ${ }^{4}$ Associate Professor, Department of Epidemiology, School of Medicine, Hamadan University of Medical Sciences, Hamadan, Iran \\ ${ }^{5}$ Associate Professor, Department of Otorhinolaryngology, School of Medicine, Hamadan University of Medical Sciences, \\ Hamadan, Iran \\ * Corresponding Author: Farnaz Hashemian, Department of Otorhinolaryngology, School of Medicine, Hamadan University \\ of Medical Sciences, Hamadan, Iran.Email: hashemian@umsha.ac.ir
}

Received: 06.04.2017

Accepted: 10.09 .2017

\section{How to Cite this Article:}

Khoshraftar E, Bakhshaei MH, Shahabinejad M, Porolajal J, Hashemian F. Comparison of the Effects of Magnesium Sulfate and Dexmedetomidine on Operation Field of Candidates for Endoscopic Sinus Surgery. Sci J Hamadan Univ Med Sci. 2017; 24(3): 177-182. DOI: 10.18869 / acadpub.ajcm.24.3.177.

\section{Abstract}

Background and Objective: One of the main treatments for chronic rhinosinusitis is endoscopic surgery. In this procedure, intraoperative bleeding due to limited view of the surgeon can bring about surgical complications. To have a clear operation field for endoscopic sinus surgery, bleeding management is necessary. We aimed to compare the effects of magnesium sulfate and dexmedetomidine on operation field of candidates for endoscopic sinus surgery.

Materials and Methods: In this triple-blind clinical trial, 60 candidates for endoscopic sinus surgery were randomly divided into two groups of Dexmedetomidine and Magnesium sulfate. Before anesthesia, magnesium sulfate was administered for $10 \mathrm{~min}$ at a dose of $50 \mathrm{mg} / \mathrm{kg}$ and at a dose 15 $\mathrm{mg} / \mathrm{kg} / \mathrm{h}$ afterwards. In the other group, dexmedetomidine was given for $10 \mathrm{~min}$ before anesthesia at a dose of $1 \mathrm{mic} / \mathrm{kg}$ and at a dose of $0.6 \mathrm{mic} / \mathrm{kg} / \mathrm{h}$ thereupon. The hemodynamic status of both groups was recorded 1, 5, 15, 30, and 60 min post-tracheal intubation. The results were analyzed using SPSS, version 16.

Results: In general, 20 (66.7\%) patients in the Dexmedetomidine group and $18(60.0 \%)$ patients in the Magnesium sulfate group were male $(\mathrm{P}=0.592)$. The mean ages of the Dexmedetomidine and Magnesium sulfate groups were 37.93 and 39.56 years, respectively $(\mathrm{P}=0.250)$. The mean surgical durations in the Dexmedetomidine and Magnesium sulfate groups were $79.03 \pm 41.8 \mathrm{~min}$ and $87.30 \pm 15.09 \mathrm{~min}$, respectively $(\mathrm{P}=0.003)$. Mean arterial pressure (MAP) in the Dexmedetomidine group was less than in the Magnesium Sulfate group at all the assessed times, except for the first time. The difference between the two groups was not significant only at first time. There was no statistical difference between the two groups in terms of mean saturation oxygen peripheral $(\mathrm{SpO} 2)$ at all the recorded times. The mean intraoperative heart rate and mean pain intensity were lower in the Dexmedetomidine group than the Magnesium sulfate group. In the Magnesium sulfate group, odds ratio of bleeding in grade 3 to grade 2 was six times higher than the Dexmedetomidine group $(\mathrm{OR}=6.00 ; 95 \% \mathrm{CI}: 1.89,19.1)$.

Conclusion: Dexmedetomidine is more beneficial for controlling hemodynamic status of sinus endoscopic surgery patients as it can provide a better operation field.

Keywords: Dexmedetomidine; Endoscopic Sinus Surgery; Magnesium Sulfate 
dof: 10.18869 /acadpub.ajcm.24.3.177

\section{مقايسه اثر سولفات منيزيوم و دكسمدتوميدين بر كيفيت فيلد جراحى در بيماران كانديد عمل جراحى اندوسكويك سينوس}

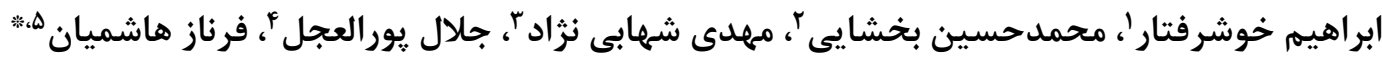

' استاديار، كروه بيهوشى، دانشكده يزشكى، دانشعاه علوم يزشكى همدان، همدان، ايران

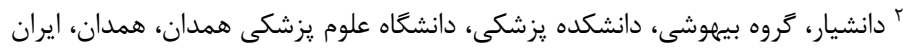

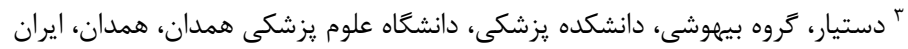

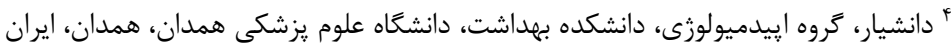

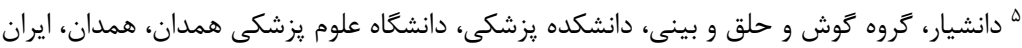
* نويسنده مسئول: فرناز هاشميان، گروه كوش و حلق و بينى، دانشكده يزشكى، دانشخاه علوم يزشكى همدان، همدان، ايران. ايميل:umsha.ac.ir

\section{جكيده}

سابقه و هدف: يكى از درمانهاى اصلى رينوسينوزيت مزمن عمل جراحى به وسيله اندوسكوبى مي باشد.

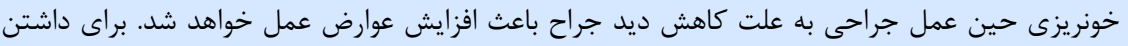

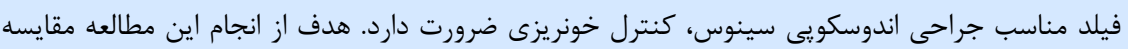

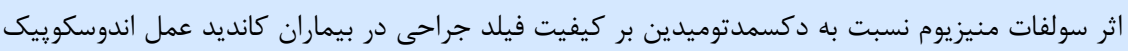
سينوس مى باشد.

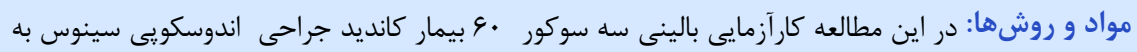

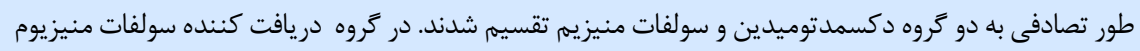

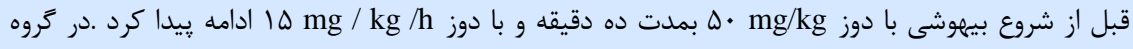
دكسمدتوميدين قبل از بيهوشى با دوز

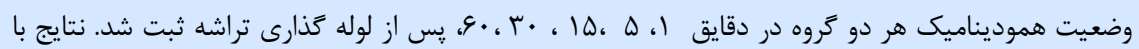

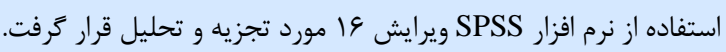

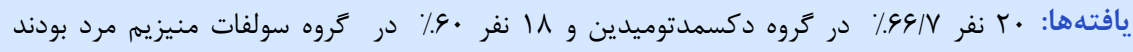

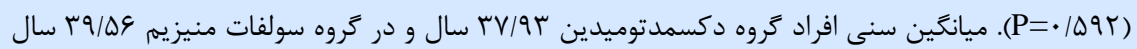

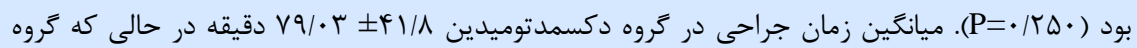

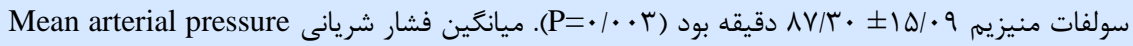

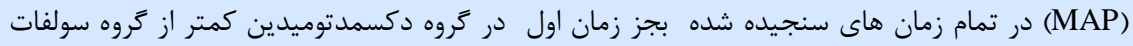

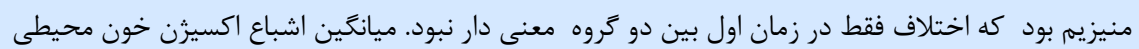
(SpO2) saturation oxygen peripheral

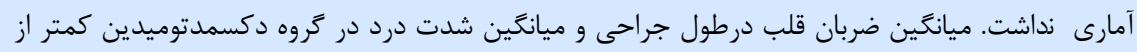

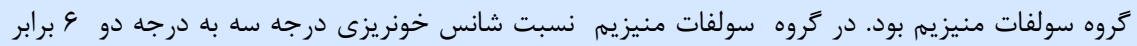

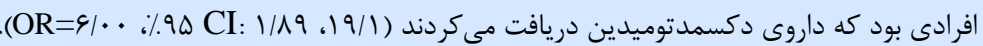

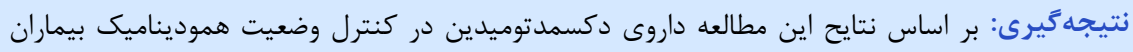

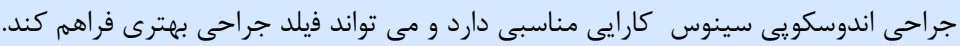
وازَّان كليدى: جراحى آندوسكوبيك سينوس؛ دكسمدتوميدين؛ سولفات منيزيم
تاريخ دريافت مقاله:

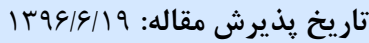

تمامى حقوق نشر براى دانشكاه علوم يزشكى همدان محفوظ است. 


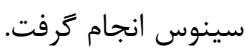

مواد و روشها

اين مطالعه بصورت يك كارآزمايى بالينى تصادفى شده سه سويه كور با كد

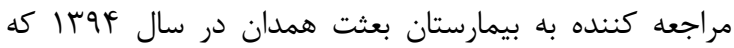

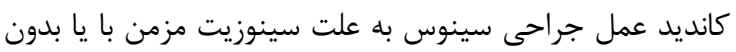

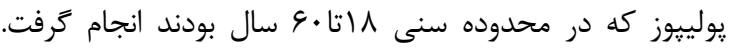
بيمارانى كه با سابقه بيمارى كليوى، كبدى، خونى ذبدى، نوروماسكولار،

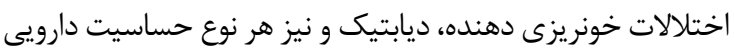

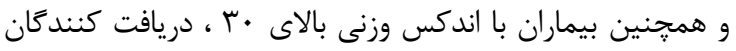

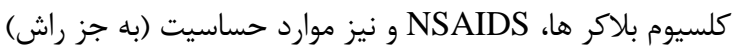
به داروهاى مورد مطالعه در اين طرح از اين مطالعه خارج شدند.

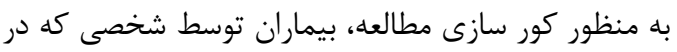

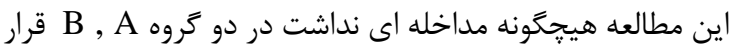

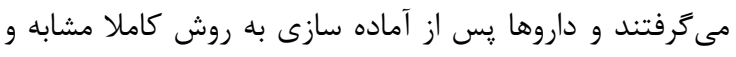
نامخذارى توسط همان فرد به همكاران اصلى مطالعه تحويل داده

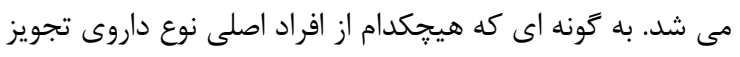

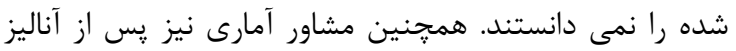

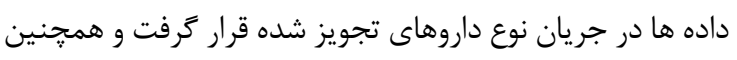

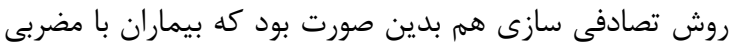

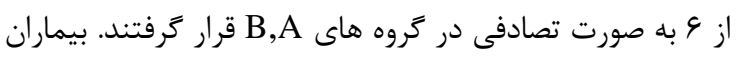

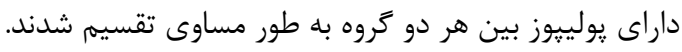

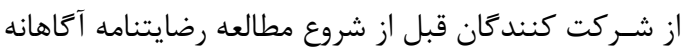

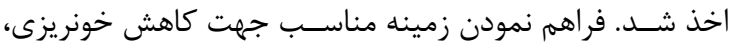

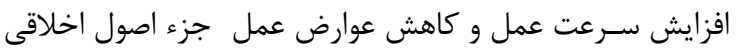

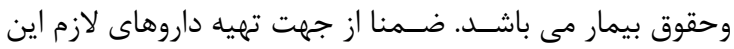

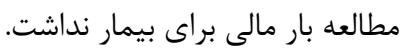

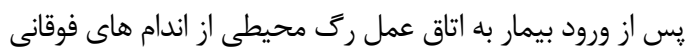

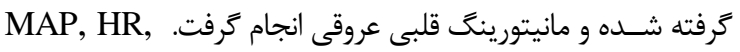

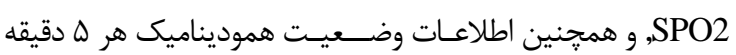

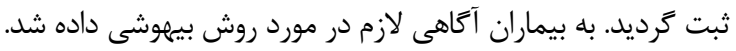

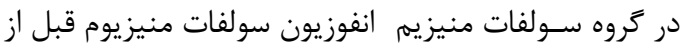

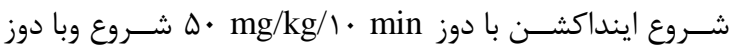

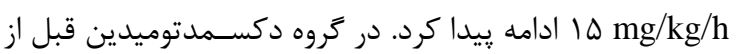

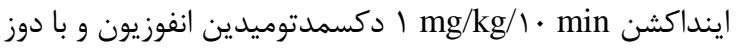

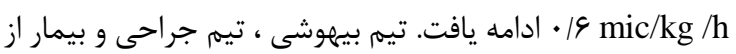

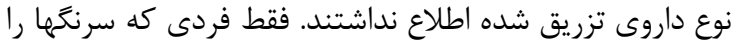

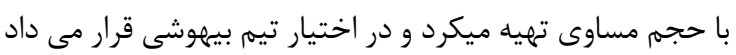

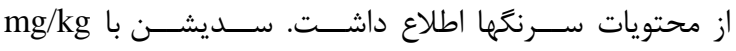

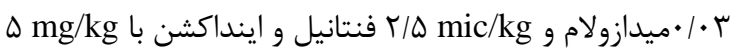

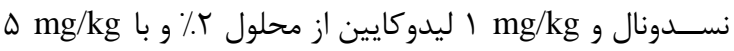
آتراكوريوم تزريق كرديد. هر يك ساعت دوز مخدر تكرار مى شد.

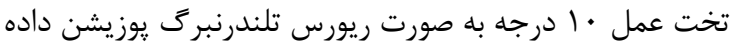

به علت كاهش ديد جراح باعث افزايش عوارض عمل خواهد شد. بنابراين كاهش خونريزى توسط متخصص بيهوشى اهميت بسيارى در جهت افزايش ديد جراح دارد. كاهش فشار خون تون كنترل شده جهت كم كردن خونريزى از نظر بهبود ديد جراح

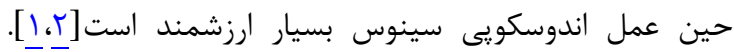

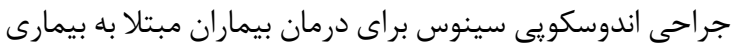

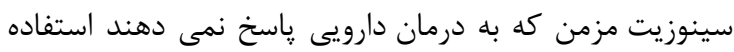

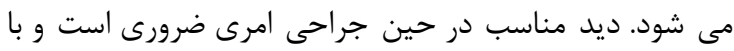

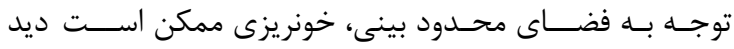

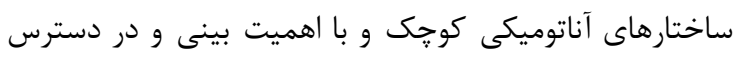
اندوسكويى را محدود كند و حتى خونريزى هاى خفيف مى تواند

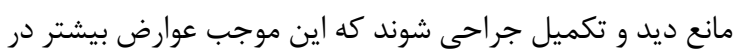
بيماران مى شود. براى كنترل فشار خون راههاى متعددى وجود دارد. هر جند

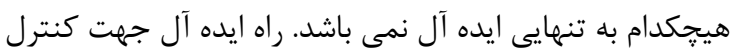

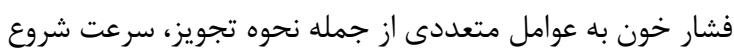

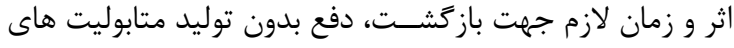
سمى در صورت بروز مشكل بستكى دارد [ـ-1_].

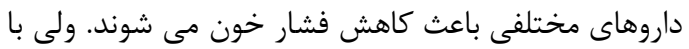

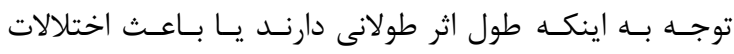

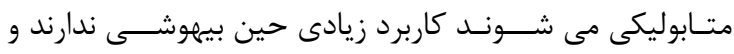

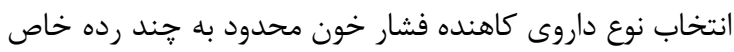

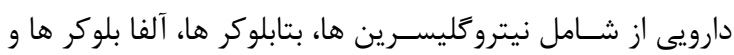
داروهاى بيهوشى مى باشد [ارئ.

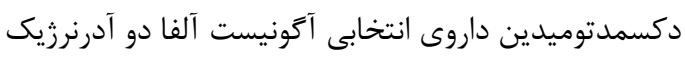

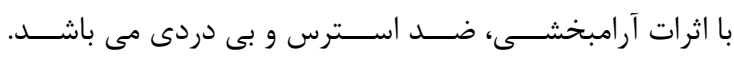

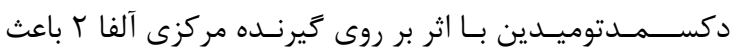

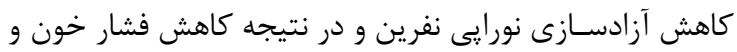
ضربان قلب مى شود [هـ].

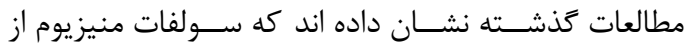
طريق فعال كردن يمب سديم يتاسيم و آنزيم كلسيم ATPase

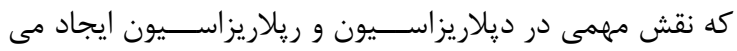

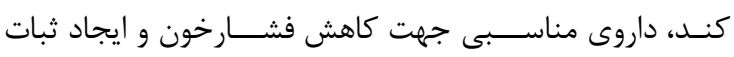

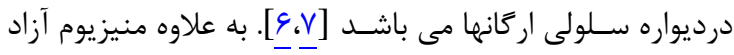

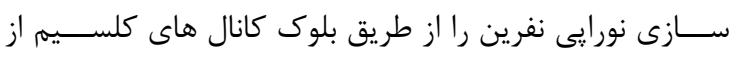

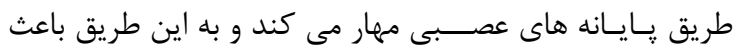

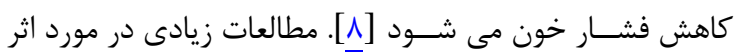

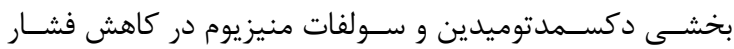
خون كنترل شـده انجام شـده است. اين دو دارو با ساير دارو ها

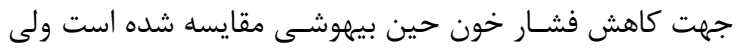

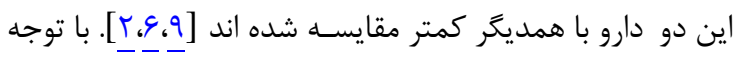

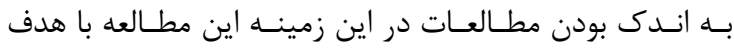

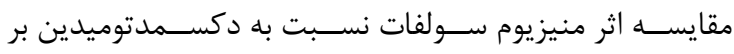

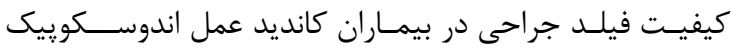


با • ب بيمار كه دكسمدتوميدين دريافت كرده بودند مقايسه شدند.

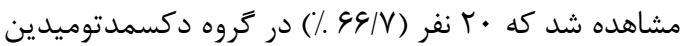

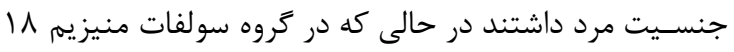

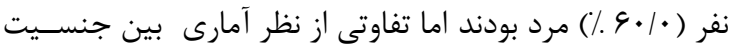

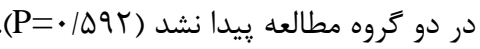

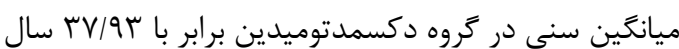

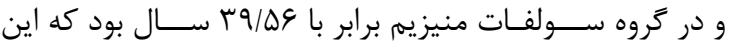

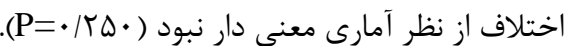

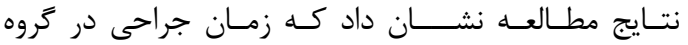

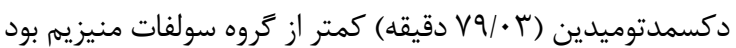

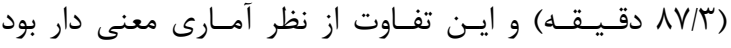
.$(\mathrm{P}=\cdot \cdot \cdot r)$

ميانگين فشار شريانى (MAP) Mean arterial pressure

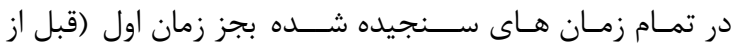
اينتوباسـيون) در گروه دكسمدتوميدين كمتر از گروه سـولفات

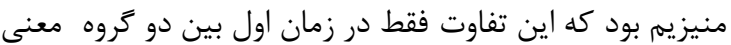

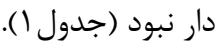

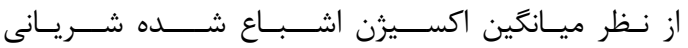

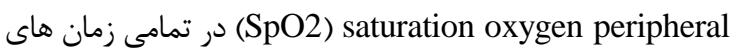
ثبـت شـــده بين دو گروه اختلاف معنى دارى بين دو گروه يِيدا

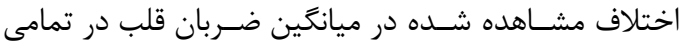
زمان هاى سنجيده شده بجز زمان اول و آخر (•

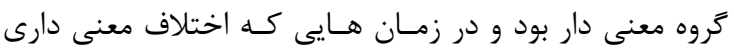

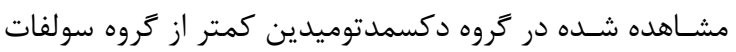

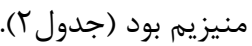

شـد و لوله تراشـهـ به وسـيله جسـب بله اطراف دهان فيكس گرديد.

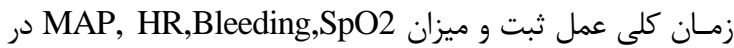

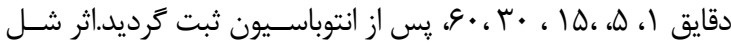

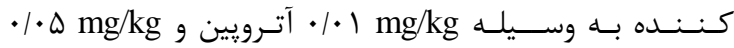

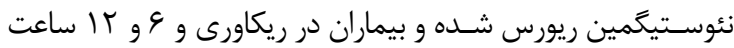

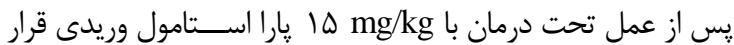

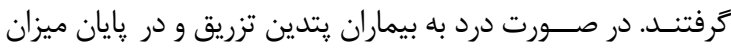

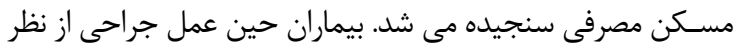
MAP , SPO2 , Bleeding

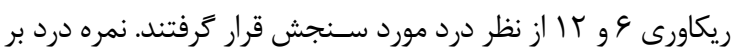
اسـاس مقياس بينايى سنجش درد (Visual Analogue Scale)

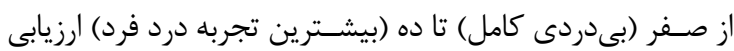

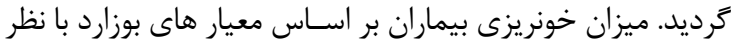

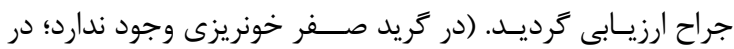

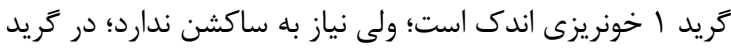

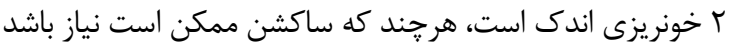

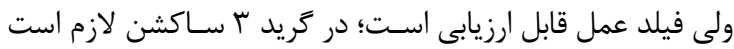

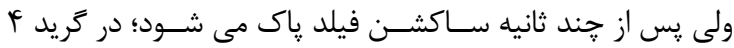

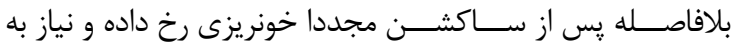

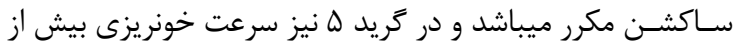
ساكشن است و عملا ادامه جراحى غير ممكن مى معن باشد).

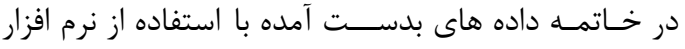
SPSS

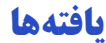
در اين مطالعه · ب بيمار كه سولفات منيزيم دريافت كرده بودند

جدول ا: مقايسه ميانكين فشار خون شريانى (ميلى متر جيوه) در زمان هاى مختلف در هر دو گروه مطالعه

\begin{tabular}{|c|c|c|c|c|c|}
\hline \multirow{2}{*}{ P(Mann-Whitney) } & \multicolumn{2}{|c|}{ سولفات منيزيم } & \multicolumn{2}{|c|}{ دكسمدتوميدين } & \multirow{2}{*}{ زمان } \\
\hline & انحراف معيار & ميانكَين & انحراف معيار & ميانگين & \\
\hline .1099 & F/or & $9 \Delta / 49$ & $F / r$ & $99 / 9$ & قبل اينتوبه \\
\hline$\cdot 1 \cdot \cdot 1$ & $V / \Delta \cdot$ & AV/Tr & $\Delta / \& \Delta$ & V\&/G. & ه دقيقه بعد \\
\hline$\cdot \cdot r$ & $1 / 19$ & $V V / T S$ & $\Delta / 11$ & $V r / f$. & ها دقيقه بعد \\
\hline$\cdot 1 \cdot \cdot 1$ & $r / 91$ & $11 / 99$ & $\varphi|q|$ & $V F / G Y$ & r r دقيقه بعد \\
\hline$\cdot 1 \cdot \cdot 1$ & f/To & $\wedge 1 / \wedge \varphi$ & $f / f \varphi$ & $V / N r$ & 9 ع دقيقه بعد \\
\hline$\cdot 1 \cdot \cdot 1$ & r/६9 & $\Lambda \mu / \kappa$. & $r / \Lambda \cdot$ & $\vee \wedge / \Delta \Lambda$ & ميانخين كل \\
\hline
\end{tabular}

جدول ץ: ميانگين ضربان قلب در دقيقه در هردو كروه مطالعه

\begin{tabular}{|c|c|c|c|c|c|}
\hline \multirow{2}{*}{ P(Mann-Whitney) } & \multicolumn{2}{|c|}{ سولفات منيزيم } & \multicolumn{2}{|c|}{ دكسمدتوميدين } & \multirow{2}{*}{ زمان } \\
\hline & انحر اف معيار & ميانتين & انحراف معيار & ميانگين & \\
\hline - IADT & $\Delta / \mathcal{F}$. & $V V / T \cdot$ & $\Delta / q \mathcal{F}$ & $\mathrm{V} / \cdot \cdot$ & قبل اينتوبه \\
\hline$\cdot 1 \cdot \cdot 1$ & $G / F V$ & $V V / q$. & $f / \Delta q$ & sq/rr & ه دقيقه بعد \\
\hline$\cdot 1 \cdot \cdot 1$ & $r / \wedge q$ & $V I / \Delta G$ & $F / T F$ & $9 \Delta / 49$ & ها دقيقه بعد \\
\hline$\cdot 1 \cdot \cdot 1$ & r/ז & SF/AG & $T / \Delta V$ & GT/r & • • ب دقيقه بعد \\
\hline$\cdot / 109$ & $F / v$ & $99 / 99$ & $f / \cdot \Delta$ & 9V/9T & و ك دقيقه بعد \\
\hline
\end{tabular}


خونريزى حين جراحى درافرادى كه داروى سولفات منيزيم

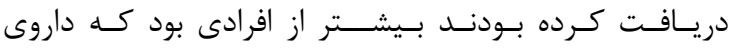

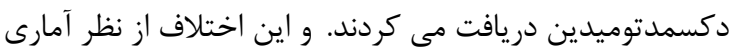

معنى دار بود. (جدول أ)
ميانكَين درد بر اســاس مقياس Visual Analog ) VAS (Scale

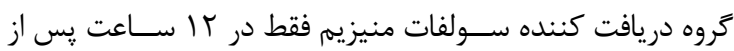
عمل كاهش معنى دارى پيدا كرد (جدول ؟).

جدول ؟ّ: مقايسه شدت درد در هر دو گروه مطالعه

\begin{tabular}{|c|c|c|c|c|c|}
\hline \multirow[t]{2}{*}{ P(Mann-Whitney) } & \multicolumn{2}{|c|}{ سولفات منيزيم } & \multicolumn{2}{|c|}{ دكسمد توميدين } & \multirow{2}{*}{ زمان } \\
\hline & انحراف معيار & ميانَين & انحراف معيار & ميانكين & \\
\hline$\cdot|99|$ & .199 & $V / F$ & $\cdot / 91$ & $V / T \&$ & ريكاورى \\
\hline $.111 \mathrm{~V}$ & .194 & ( & $\cdot / \Delta \Delta$ & $\mathrm{F} / \mathrm{\Lambda}$. & 9 ساعت بعد \\
\hline$\cdot / \cdot r$ & .191 & $F / F Q$ & $1 / \cdot r$ & ए/९६ & Tا ساعت بعد \\
\hline
\end{tabular}

جدول F): مقايسه گريد خونريزى در هر دو گروه مطالعه

\begin{tabular}{|c|c|c|c|}
\hline $\mathbf{P}\left(\operatorname{chi}^{2}\right)$ & فراوانى (درصات منيزيم) & فركسمدتوميدين & خونريزى \\
\hline \multirow{3}{*}{$\cdot 1 \cdot r$} & $(r \cdot / \cdot)^{9}$ & $(9 \cdot / \cdot) 11$ & كريد r \\
\hline & $(\Lambda \cdot / \cdot) T^{F}$ & $(k \cdot / \cdot)$ IT & گريد \\
\hline & $(1 \cdot \cdot / \cdot) \mu \cdot$ & $(1 \cdot \cdot / \cdot) \Gamma \cdot$ & جمع \\
\hline
\end{tabular}

ساير داروها باشد[ب] كه با نتايج مطالعه ما همسو مى باشد.

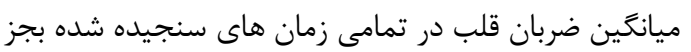

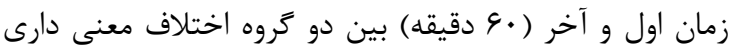

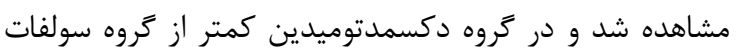

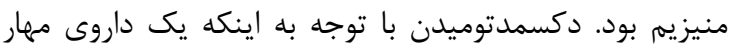

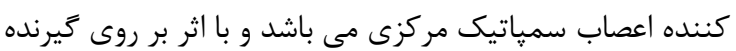

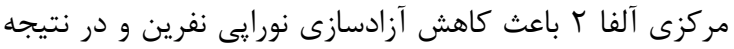
كاهش فشار خون و ضربان قلب مى شود [1 I [1].

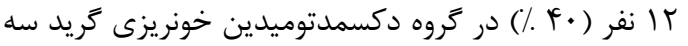

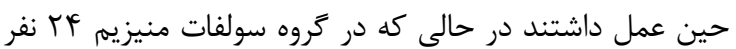

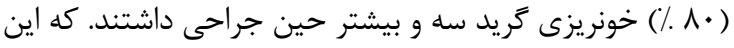

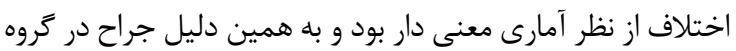

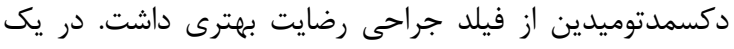

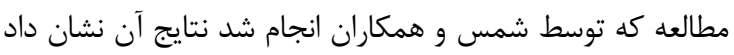

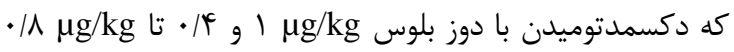
انفوزيون كارايى مناسبى براى فيلد جراحى دارد [ـإ] كه با نتايج مطالعه حاضرهمسو مى باشد. درمطالعه ى ظريف و همكاران نشان

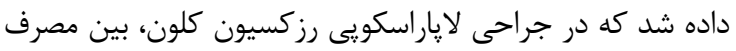

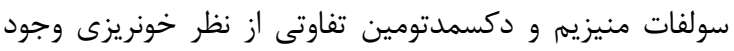

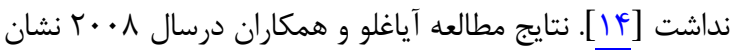

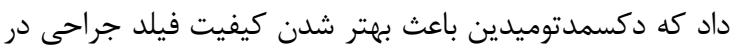
جراحى گوش مى شود و اين بيماران نيازمند ميزان كمترى فنتانيل

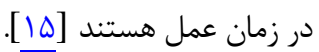

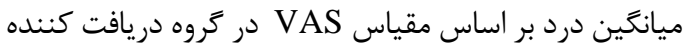

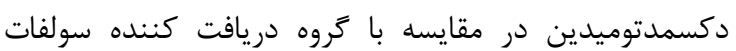

هدف از انجام اين مطالعه مقايسه اثر سولفات منيزيوم نسبت

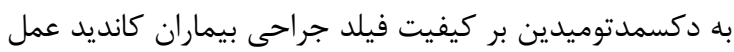
جراحى اندوسكوييك سينوس بود.

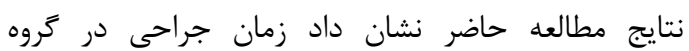

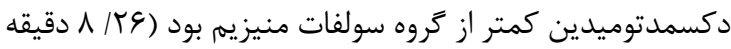

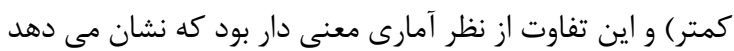

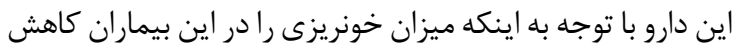

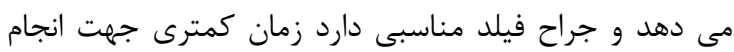

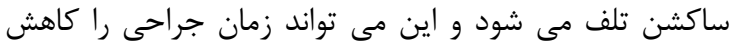

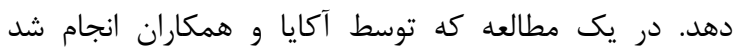

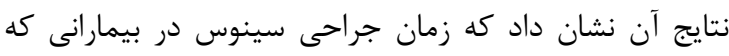

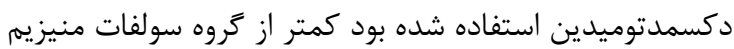

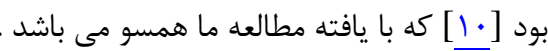

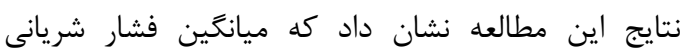

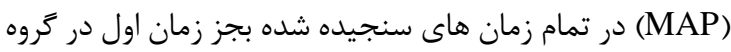

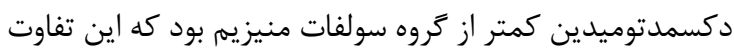

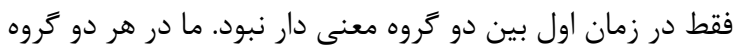

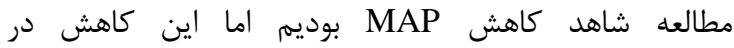
دكسمدتومين بيشتر از سولفات منيزيم بود. نتايج مطالعه

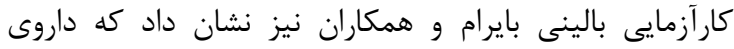

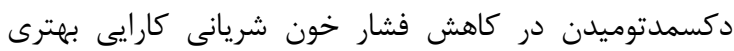

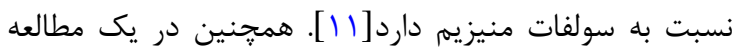

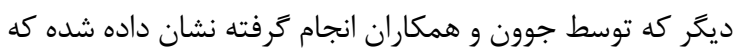

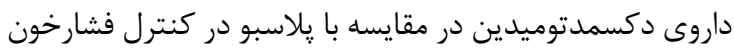

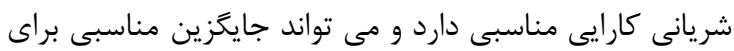




$$
\begin{aligned}
& \text { وضعيت هموديناميك بيماران جراحى اندوسكويى سينوس }
\end{aligned}
$$

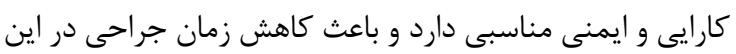

$$
\begin{aligned}
& \text { بيماران مى شود. همجنين براساس يافته هاى مطالعه حاضر }
\end{aligned}
$$

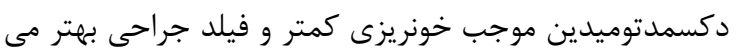

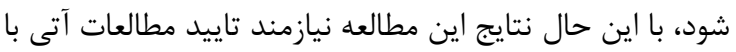

$$
\begin{aligned}
& \text { حجم نمونه بزركتر است. } \\
& \text { تشكر و قدر فاذى } \\
& \text { اين مقاله بر گرفته از يايان نامه دوره دستيارى مى باشد و }
\end{aligned}
$$

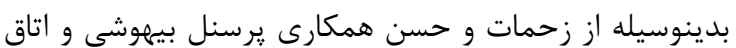

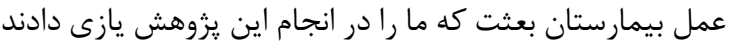

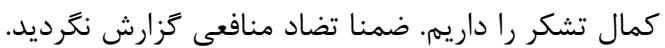

\section{REFERENCES}

1. Degoute CS. Controlled hypotension: a guide to drug choice. Drugs. 2007;67(7):1053-76. PMID: 17488147

2. Guven DG, Demiraran Y, Sezen G. Evaluation of outcomesin patients given dexmedetomidine in functional endoscopicsinus surgery. Ann Otol Rhinol Laryngol. 2011;120(9):586-92. PMID: 22032072 DOI: $10.1177 / 000348941112000906$

3. Marchal JM, Gomez-Luque A, Martos-Crespo F. Clonidinedecreases intraoperative bleeding in middle ear microsurgery. Acta Anaesthesiol Scand. 2001;45(5):627-33. PMID: 11309017

4. Choi HA, Ko SB, Presciutti M, Fernandez L, Carpenter AM, Lesch $\mathrm{C}$, et al. Prevention of shivering during therapeutic temperature modulation: the Columbia anti-shivering protocol. Neurocrit Care. 2011;14(3):389-94. PMID: 21210305 DOI: $10.1007 / \mathrm{s} 12028-010-9474-7$

5. Jooste EH, Muhly WT, Ibinson JW, Suresh T, Damian D, Phadke A, et al. Acute hemodynamicchanges after rapid intravenous bolus dosing of dexmedetomi-dine in pediatric heart transplant patients undergoing routinecardiac catheterization. Anesth Analg. 2010;111(6):1490-6. PMID: 21059743 DOI: 10.1213/ANE.0b013e3181f7e2ab

6. Ryu JH, Sohn IS, Do SH. Controlled hypotension for middle earsurgery: a comparison between remifentanil and magnesiumsulfate. Br J Anaesth. 2009;103(4):490-5. PMID: 19687032 DOI: $10.1093 /$ bja/aep229

7. Koinig H, Wallner T, Marhofer P, Andel H, Hörauf K, Mayer $\mathrm{N}$. Magnesium sulfate reducesintra- and postoperative analgesic requirements. Anesth Analg. 1998;87(1):206-10. PMID: 9661575

8. Shimosawa T, Takano K, Ando K. Magnesium inhibitsnorepinephrine release by blocking $\mathrm{N}$-type calcium chan-nels at peripheral sympathetic nerve endings. Hypertension. 2004;44(6):897-902. PMID: 15477382 DOI: 10.1161/01.HYP.0000146536.68208.84

9. Elsharnouby NM, Elsharnouby MM. Magnesium sulphate as a technique of hypotensive anesthesia. Br J Anaesth. 2006;96(6):727-31.

$$
\begin{aligned}
& \text { منيزيم فقط در r| ساعت يس از عمل كاهش معنى دارى پيدا }
\end{aligned}
$$

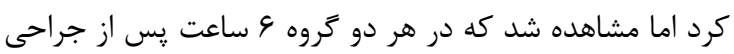

$$
\begin{aligned}
& \text { نيز كاهش قابل ملاحظه اي ييدا كرد. } \\
& \text { اثر ضد دردى دكسمدتوميدن در مطالعات مختلف نشان داده مليد }
\end{aligned}
$$

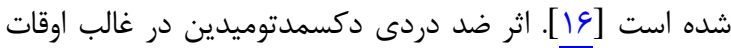

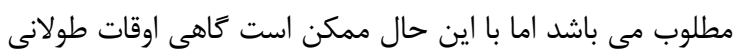

$$
\begin{aligned}
& \text { شود. در مطالعه شمس و همكاران نشان داده شد كه استفاده از }
\end{aligned}
$$

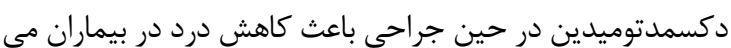

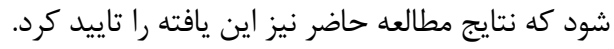

$$
\begin{aligned}
& \text { نتيجه تيرى } \\
& \text { بر اساس نتايج اين مطالعه داروى دكسمدتوميدين در كنترل }
\end{aligned}
$$

10. Akkaya A, Tekelioglu UY, Demirhan A, Bilgi M, Yildiz I, Apuhan T, et al. Comparison of the effects of magnesium sulphate and dexmedetomidine on surgical vision quality in endoscopic sinus surgery: randomized clinical study. Rev Bras Anestesiol. 2014;64(6):406-12. PMID: 25437697 DOI: 10.1016/j.bjan.2014.01.008

11. Bayram A, Ulgey A, Gunes I, Ketenci I, Capar A, Esmaoglu A, et al. Comparison between magnesium sulfate and dexmedetomidine in controlled hypotension during functional endoscopic sinus surgery. Rev Bras Anestesiol. 2015;65(1):617. PMID: 25497751 DOI: $10.1016 /$ j.bjan.2014.04.003

12. Bryskin R, Weldon BC. Dexmedetomidine and magnesium sulfate in the perioperative management of a child undergoing laparoscopic resection of bilateral pheochromocytomas. J Clin Anesth. 2010;22(2):126-9. PMID: 20304355 DOI: 10.1016/j.jclinane.2009.01.017

13. Shams T, El Bahnasawe NS, Abu-Samra M, El-Masry R. Inducedhypotension for functional endoscopic sinus surgery: a comparative study of dexmedetomidine versus esmolol. Saudi JAnaesth. 2013;7(2):175-80. PMID: 23956719 DOI: 10.4103/1658-354X.114073

14. Zarif P, Abdelaal Ahmed Mahmoud A, Abdelhaq MM, Mikhail HM, Farag A. Dexmedetomidine versus magnesium sulfate as adjunct during anesthesia for laparoscopic colectomy. Anesthesiol Res Pract. 2016;2016:7172920. PMID: 27051421 DOI: $10.1155 / 2016 / 7172920$

15. Ayoglu H, Yapakci O, Ugur MB, Uzun L, Altunkaya H, Ozer $\mathrm{Y}$, et al. Effectiveness ofdexmedetomidine in reducing bleeding during septoplasty andtympanoplasty operations. $J$ Clin Anesth. 2008;20(6):437-41. PMID: 18929284 DOI: 10.1016/j.jclinane.2008.04.008

16. Huncke TK, Adelman M, Jacobowitz G, Maldonado T, Bekker A. A prospective, randomized, placebo-controlled study evaluating the efficacy of dexmedetomidine for sedation during vascular procedures. Vasc Endovascular Surg. 2010;44(4):257-61. PMID: 20308169 DOI: $10.1177 / 1538574410363621$ 